\title{
Neutropenia previa al tratamiento quimioterápico en leucemia linfática aguda
}

\author{
Hugo García U.', 2; Lautaro Vargas P. ${ }^{1,2}$, Carmen Layte V. \\ Neutropenia and fever at initial presentation \\ of acute lymphocytic leukemia
}

\begin{abstract}
Amorn 42 patients with acute lymphocytic leukernia $\{A L L\}$ under control at a metropolitan teaching hospital from Sartiago, Chile, $34(81 \%)$ had initiai granulocyte counts under 1500 per $\mathrm{mm}^{3}$ and 26 [62\%) under 500 nes. trophyls per $\mathrm{mm}^{3}$ isevere 7 eutropenia). Thirteen patients of the whole (30,9\%) had fever cr presentation, and 12 of these had severe or extreme \{granulocyte count under 100 per $\mathrm{mm}^{3}$ \} neutropenia. Nine in 12 patients with fever and severe or extreme neutropenia had clinical or $X$ ray evidence of an infectious focus $475 \%$, which in six cases was at the respiratory tract and in three $(25 \%$ ) gave positive bloot culture results (Staphylococcus aureus in two patients with pneumonia and Klebsiella so. in a case with upper lip abscess). With some minor exceptions all these children were initially treated bv an association of penicilin, cioxacillin and gentamycin accordingly to local Experience for empirical treatment - this was modified only when justified by clinical course or bacteriological results- with onfy one death $(B, 3 \%)$ among them. Six of 14 non febrile neutropenic patients had s,oecific clinical infections. which in three gave positive culture results (two patients with urirary Escherichia coli infections and Pseudomonas aeruginosa from a girl with an abscess of the external genitalia). thes were treated only for the spe. cifir clinical situation in accordance with bacterial sensitivity and no one died. Antilaukanic induction therapy swas initiated in most cases before 10 days from initial symptoms.
\end{abstract}

(Key words: Leukemia, acute lympnocytic, neutropenia, infections, treatment.)

Cuando hay neutropenia y el número de granulocitos es inferior a 500 por $\mathrm{mm}^{3}$, ella es el factor más importante entre los que favorecen las infecciones bacterianas $y$ por hongos de los enfermos con cáncer ${ }^{1}$. En la mayoría de las enfermedades oncológicas aparece como complicación de la quimioterapia. En los pacientes con leucemia aguda puede existir desde el inicio de la enfermedad, formando parte del sindrome de insuficiencia medular caracteristico de la enfermedad $y$, tanto la forma linfática como la mieloide, pueden manifestarse inicialmente, en una proporción importante de enfernos, con neutropenia asociada o no a fiebre, con 0 sin in. fección detectable. Si hay neutropenia $y$ el número de neutrófilos es inferior a $500 \times \mathrm{mm}^{3}$ y se acompaña de fiebre debe indicarse precoz-

1. Servicio de Pediatría. Hospital San Juan de Dios, Unidad Hematouncología,

2. Departamento Pediatría y Cirugía Infantil, Dívisión Ciencias Médicas Occidente. Facultad de Medicina, Universidad de Chile. mente antibióticos de amplio espectro, sin esperar resultados bacteriológicos, asumiendo que el enfermo está infectado, aunque en algunos casos la fiebre puede explicarse por el propio proceso neoplásico. Este tratamiento empírico ha contribuido a disminuir significativamente la letalidad en enfermos de cancer ${ }^{2}$. En la mayoría de los casos es el pediatra general quien descubre la leucemia aguda y debe estar preparado para enfrentar con éxito las infecciones iniciales de los enfermos con leucemia antes de derivarlos, pues de la rapidez en identificarlas y tratarlas adecuadamente dependerá el pronóstico vital de estos niños.

A continuación se analiza la neutropenia injcial en pacientes con leucemia linfática aguda (L.LA) con los propositos de: describir su frecuencia, su relación con signos clínicos y evidencia bacteriológica de infecciones, su repercusión sobre el inicio de la terapia de inducción, la evolución de estos niños y la utilidad del tratamiento precoz y empírico con antibióticos de amplio espectro. 


\section{Material y Método}

Entre los años 1984 y 1989 se han estudiado $42 \mathrm{ca}-$ sos evaluables de LLA en la Unidad de Hematología y Oncología Infantil del Hospital San Juan de Dios, los que, para efectos de este estudio, fueron analizados de acuerdo al número absoluto de neutrófilos en el hemograma del diagnóstico. La meutropenia se consideró severa si el recuento absoluto de neutrófilos era interior a 500 , extrema si era inferior a $100 \cdot \mathrm{mm}^{3}$, moderada si había entre 500 y 1000 neutrófilos $\cdot \operatorname{mon}^{3}$ y leve con 1001 a 1500 neutrófios. Se definió fiebre por tem. peratura axilar de $39^{\circ} \mathrm{C}$ en una medición o de $38,5^{\circ} \mathrm{C}$ en dos oportunidades separadas por cuatro horas sin haber mediado transfustón de algún hemoderivado. En los pacientes con neutropenia severa y extrema se hizo an examen físico particularmente protijo, espccialmente en sitios puerta de entrada de infecciones como piel, mucosas y zonas periorificiales; se tomaron muestras para tres hemoculitivos, cultivo de secreción tar ingea, orina y deposiciones, incluyendo siembras para gérmenes anaerobios, cuando se considetó necesario, de algunas zonas sospechosas. Por la estrecha asociación de neutropenia febrit e infección respiratoria se solicitó radiografía de tórax en todos los casos, que fueron aisiados en piezas individuales durante su permanencia en el hospital.

\section{Resultados}

Entre 42 pacientes con LLA, $26(61,9 \%)$ tenían neutropenia extrema (n: $9: 21,4 \%, 6$ de éstos con fiebre) o severa (n: $17 ; 40,5 \%, 6$ de ellus también con fiebre) en el hemograma inicial: cuatro $(9,5 \%$, ninguno con fiebre) tenian neutropenja moderada, otros cuatro neutropenia leve (uno tenía fiebre), mientras el recuento de neutrófilos era normal en ocho casos (19\%, uno con fiebre). En suma, 13 de 42 pacientes con LLA $(30,9 \%)$ tenían neutropenia febril, 12 de los cuales correspondian a 26 casos $(46,1 \%)$ con neutropenia severa o extrema y sólo uno a los ocho pacientes con neutropenia leve a mode. rada. Los niños con recuento normal de neutrófilos eran en su mayoría enfermos que presentaban inicialmente hiperleucocitosis sobre 100000 por $\mathrm{mm}^{3}$.

Nueve de los 12 casos con fiebre y neutrope. nia severa $(75 \%)$ tenian evidencia de un foco infeccioso al examen clínico, radiológico o ambos: en cinco el resultado bacterjológico fue negativo, en 3 los hemocultivos fueron positivos (25\%) y el coprocultivo fue positivo en un caso de enterocolitis aguda. La fiebre como único signo se presentó en 3 casos $(25 \%)$. E] foco infeccioso predominante fue respiratorio (en cuatro casos neumopatía aguda; en dos, infecciones respiratorias de la vía aérea superior). Los hemocultivos fueron positivos para $S t a-$ phylococcus aureus en dos niños con bronconeumonia y Klebsiella en uno con flegmón del labio superior. En 9 de los 12 niños se decidió indicar una asociación antibiótica de amplio espectro, incluyendo la precaución de administrar antibióticos a todos los pacientes febriles con neutropenia extrema, sin considerar la presencia a ausencia de un foco infeccioso En ocho se indicó la asociación de penicilina. cloxacilinaugentamicina como combinación de primera linea, a las dosis habituales, que siete recibieran por de 10 a 14 dias, el otro sufría sepsis a Klebsiella y al cabo de tres dias el esquema se cambió por la asociación de cefalosporina de tercera generación y amikacina, tam. bjén por 14 días. En el paciente con diagnóstico clínico de sepsis, dada la gravedad de su cuadro (Tiebre choque y compromiso general severo) se usó desde el comienzo la asociación de cefradina-cloxacilina y amikacina hasta completar 14 días. En tres niños no se usaron antibióticos, pues la fiebre duró sólo 24 horas y se habia decidido observarlos, dos no tenían foco detectable y la bacteriologia dio resultados nega. tivos, el tercero tenía evidencia clínica de farin. gobronquitis aguda viral (tabla 1). La evolución fue favorable en la mayoría de los pacientes, la fiebre descendió en 3 a 5 días, salvo en el niño con sepsis clínica en que duró más de 7 días. El paciente con neumonía lobar y ausencia de neutrófilos en el recuento, falleció al cuarto día de evolución. En este caso no se realizó estudio anatomopatológico. De este modo, la letalidad para todo el grupo neutropénico febril severo fue de $8,3 \%$, pero si se consideran sólo los casos de neutropenia extrema la letalidad sube a $16,6 \%$ (un fallecido en seis casos).

Considerando que la única manera de revertir la neutropenia era comenzas precozmente la quimioterapia, en 11 de los 12 pacientes con neutropenia febril severa se inició đicho tratamiento entre los 5 y 9 días de evolución intrahospitalaria, cuando el síndrome febril habia ceủdo y aún recibian antibióticos. En el niño con sepsis clínica, cuya evolución febril fue arrastrada, la quimioterapia se inició a los 16 días de evolu. ción.

En la tabla 2 se resumen las características clinicas, bacteriológicas y el tratamiento efectuado en los 14 pacientes con neutropenia severa afebril, sólo tres de los cuales mostraban neutro- 
Tabla 1

Relación entre neutropenia febril severa foco infeccioso, bacteriología y tratamiento antibiótico

\begin{tabular}{|c|c|c|c|c|c|}
\hline $\begin{array}{l}\text { Neutrófilos } \\
\text { (n) }\left(\mathrm{mm}^{3}\right)\end{array}$ & $\begin{array}{c}\text { Foco } \\
\text { infeccioso }\end{array}$ & $\begin{array}{c}\text { Estudio } \\
\text { bacteriológico }\end{array}$ & \multicolumn{3}{|c|}{$\begin{array}{l}\text { Asociación } \\
\text { antibióticos }\end{array}$} \\
\hline 0 & $\begin{array}{l}\text { Branconcumon ia- } \\
\text { enterocolitis }\end{array}$ & $\begin{array}{l}\text { Coprocultivo: C. Albicans, } \\
\text { É. coli enteroparógena }\end{array}$ & $P+C+G+K$ & $x$ & 14 días \\
\hline 0 & Flegmón labio superior & $\begin{array}{l}\text { Hemocultivo: Xlebsiella } \\
\text { Cultivo labial: E. aureus }\end{array}$ & $\begin{array}{l}\mathrm{P}+\mathrm{C}+\mathrm{G} \\
\mathrm{CF}+\mathrm{A}+\mathrm{N}\end{array}$ & $\begin{array}{l}x \\
x\end{array}$ & $\begin{array}{l}3 \text { días } \\
14 \text { días }\end{array}$ \\
\hline 0 & Neumonía lobular & Negativo & $P+C+G$ & $\mathrm{x}$ & 4 dias \\
\hline 0 & Amigdalit is pultácea & Negativo & $P+C+G$ & $\mathrm{x}$ & 14 días \\
\hline 0 & $\operatorname{Sin}$ foco infeccioso & Negativo & $P+C+G$ & $\mathrm{x}$ & 14 dias \\
\hline 26 & Abdomen agudo operado & Negativo & $P+C+G$ & $\mathrm{x}$ & 14 dias \\
\hline 120 & Sin foco infeccioso & Negativo & \multicolumn{3}{|c|}{ Sin antibióticos } \\
\hline 124 & Sepsis clinica & Negativo & $\mathrm{CF}+\mathrm{A}+\mathrm{C}$ & $\mathrm{x}$ & 14 días \\
\hline 130 & Sin foco infeccioso & Negativo & \multicolumn{3}{|c|}{ Sin antibióticos } \\
\hline 360 & Faringobronquitis & Negatiro & \multicolumn{3}{|c|}{ Sin antibióticos } \\
\hline 424 & Bronconcumonía & Hemocultivo: $E$. aureus & $\mathrm{P}+\mathrm{C}+\mathrm{C}$ & $\mathrm{x}$ & 14 dias \\
\hline 429 & Bronconeumonía & Hemocultivo: E. oureus & $\mathrm{CF}+\mathrm{A}+\mathrm{C}$ & $x$ & 14 dias \\
\hline $\begin{array}{l}\text { P: penicilina } \\
\text { C: cloxacilina }\end{array}$ & $\begin{array}{l}\text { G: gentamicina } \\
\text { CF: cefradina }\end{array}$ & $\begin{array}{l}\text { A: amikacina } \\
\mathrm{N} \text { : nistalina }\end{array}$ & \multicolumn{3}{|c|}{ K: ketonazol } \\
\hline
\end{tabular}

penia extrema. En ocho de estos casos no se demostró al examen físico un foco infeccioso, su estudio bacteriológico fue negativo y todos quedaron en observación, sin antibióticos. En los otros seis se jdentificó un problema clínico específico, en tres los exámenes bacteriológicos fueron positivos (dos infecciones urinarias con urocultivo positivo y un flegmón del labio mayor con cultivo de secreción positivo a Pseudomonas aeruginosa. Todos ellos fueron tratados con los antibióticos adecuados, con buena respuesta clínica, sin letalidad ni fiebre en el curso de la evolución previa a la quimioterapia, cuya fase de inducción se inició en la totalidad de los casos antes de los 7 dias de hospitalización.

\section{Comentario}

La neutropenia forma parte del cuadro inicial de insuficiencia medular en la LLA, sien- do frecuente también en la fase inicial, como en la serie que analizamos, donde era severa en más de la mitad de los pacientes. En pacientes leucémicos que han recibido tratamiento se ha demostrado relación entre el número de neutrófilos y la susceptibilidad a las infecciones, cuyo riesgo se eleva considerablemente cuando el número de neutrófilos desciende de $\mathbf{5 0 0}$ por milimetro cúbico ${ }^{1-3}$. Alrededor del $30 \%$ de los pacientes con neutropenia severa presentan infecciones versus $5 \%$ si el recuento absoluto de neutrófilos (RAN) es mayor de $500^{2}$. La incidencia de bacteremias e infecciones severas es mayor aún si el RAN es inferior a 100 neutrófilos ${ }^{1,4,5}$. La quimioterapia más agresiva, especialmente en las fases de inducción y consolidación, produce neutropenias intensas y prolongadas, a las que se agregan otros factores que favorecen $y$ agravan las infecciones, como la colonización por gérmenes intrahospitalarios con resistencia a los antibióticos 6,7 , la común presencia de mucositis 
Tabla 2

Relación entre neutropenia afebril severa foco infeccioso, bacteriología y tratamiento antibiótico

\begin{tabular}{|c|c|c|c|}
\hline $\begin{array}{l}\text { Neutrófilos } \\
\text { (n) }\left(\mathrm{mm}^{3}\right)\end{array}$ & $\begin{array}{c}\text { Foco } \\
\text { infeccioso }\end{array}$ & $\begin{array}{c}\text { Estudio } \\
\text { bacteriológico }\end{array}$ & $\begin{array}{l}\text { Asociación } \\
\text { antibióticos }\end{array}$ \\
\hline 0 & Sin foco infeccioso & Negativo & No \\
\hline 40 & Infección urinaria & Urocultivo: $E$. colt & $G \times 10$ dias \\
\hline 31 & Sin foco infeccioso & Negativo & No \\
\hline 165 & Sin foco infeccioso & Negativo & No \\
\hline 180 & Bronconeumonía & Negativo & $P \times 10$ días \\
\hline 240 & Sin foco infeccioso & Negativo & No \\
\hline 264 & Sin foco infeccioso & Negativo & No \\
\hline 280 & Flegmón labio mayor & $\begin{array}{l}\text { Cultivo secreción: } \\
\text { P. aeruginosat }\end{array}$ & CF $2 \times 14$ días \\
\hline 320 & Sin foco infeccioso & Hemocultivo serratia & No \\
\hline 336 & Diatrea aguda & Negativo & $G \times 6$ dias \\
\hline 356 & Infección urinaria & Urocultivo: $E$ coll & $\mathrm{G} \times 10$ días \\
\hline 420 & Sin foco infeccioso & Negativo & No \\
\hline 440 & Bronconeumonía & Negativo & $P \times 10$ días \\
\hline 454 & Sin foco infeccioso & Negativo & No \\
\hline P: penicilina & G: gentamicira & CFZ: ceftazidina & \\
\hline
\end{tabular}

causada por algunos quimioterápicos y los procedimientos invasivos de tratamiento de estos enfermos, que muchas veces implican romper la barsera de la piel o usar catéteres, entre otros factores que se suman para favorecer las infecciones $5,8,9$.

La fiebre y la infección son muy frecuentes en la presentación inicial de la LLA, variando su incidencia entre 15 y $61 \%^{10-12}$, entre los que se ubican nuestros resultados, confirmando esta aseveración y la importancia de considerat la fjebre y la neutropenia, en la LLA, un sintoma alarmante que requiere buscar la localización de la infección, hacer su identificación microbiológica e iniciar el tratamiento. Nuestra casuística demuestra que la neutropenia es el factor más importante en la complicación infecciosa y en la gravedad de ella, ya que el único pacien. te fallecido correspondió a un enfermo con neutropenia extrema y neumonia lobar. En grandes casuísticas de pacientes oncológicos con neutropenia la fiebre es el único signo reconocible de infección en 30 ó 40 de los casos, sólo en 30 a $40 \%$ se logra detectar un foco de infección por medios clínicos y sólo en 20 a 30\% de los afectados es posible obtener hemocultivos positi$\operatorname{vos}^{5,15}$

La microbiología de las infecciones en los enfermos con cáncer corresponde en $85 \%$ de ellas a bacterias, cuya identidad ha cambiado a través del tiempo. Entre 1950 y 1960 predominaban los gérmenes Gram positivo, especialmente el Staphylococcus aureus. Entre 1960 y 1980 la mayoria eran Gram negativo, actualmente han reaparecido los Gram positivo como Staphylococcus epidermis y otras bacterias oportunistas. 
Nuestros hallazgos en esta materia coinciden con los de otros autores nacionales ${ }^{16}$.

Los episodios neutropénicos febriles de la LLA previos al tratamiento quimioterápico no parecen diferir de los causados por quimioterapia, por lo que se debe tomar la nisma condueta que en éstos, indicando un tratamiento antibiótico empírico que debe ser precoz, de amplio espectro y sin esperar el resultado bacteriológico. Antes de apliçar dicho criterio empírico de manejo, la letalidad por infecciones alcanzaba proporciones cercanas al $80 \%$, especialmente en la sepsis a Gram negativo, en que los enfermos fallecian de manlera fulminante en las primeras horas de evolución del cuadro febril ${ }^{5}: 17-19$. Con el tratamiento antibiótico empírico precoz la mortalidad ha descendido espectacularmente ${ }^{20,21}$.

E1 esquema antibiótico debe cumplir con los requisitos indispensables de incluir drogas bactericidas, que cubran el mayor espectro, de acuer. do a la microbiologia local y baja toxicidad. Se han recomendado múltiples combinaciones de un aminoglicósido con una cefalosporina, o un aminoglicósido y dos betalactámicos $\mathrm{y}$, por último, monoterapia con ceftazidina ${ }^{22}$, sugerencia esta última que no se ha impuesto, ya que en la experiencia del propio autor de ella, fue necesario agregar otros antibióticos en $42 \%$ de los casos. Si la neutropenia se prolonga, la necesidad de modificar la nonoterapia alcanza al $67 \%$. Por otra parte, la emergencia de Staphylococcus epidemis ha hecho que algunos expertos, especialmente de EUA, agreguen vancomicina a los esquemas clásicos. En nuestra unidad, hasta 1989, hemos usado una combinación de penicilina, gentamicina y cloxacilina, si el enfermo no había estado hospitalizado recientemente. Si la temperatura no descendía en 72 horas, se cambiaba penicilina por una cefalosporina de tercera generación y gentamicina por amikacina. Cuan. do hay neutropenia extrema, se indica ceftazi. dina más amikacina por la alta probabilidad de infecciones debidas a Pseudomonas aenuginosa. Śilo en tres casos se difírió el uso de antibióticos, debido a que la fiebre duró sólo algunas horas. En general, la respuesta clínica fue satis. factoria ocurriendo tan sólo la muerte ya mencionada, en un paciente con neutroperia extrema febril, caso en que es recomendable usar el tratamiento antibiótico más agresivo. Afortunadamente no ocurrieron infecciones sistémicas con hongos, más frecuentes cuando la neutro- penia se prolonga en el tiempo. En los casos en que se detectaron reservorios de Candida albicans fueron tratados con nistatina o ketoconazol.

\section{Resumen}

Se analiza la neutropenia inicial en 42 casos de LLA evaluados en la Ĺnjdad de Oncohematología del Hospital San Juan de Dios entre los años 1984 y 1989 . En el $81 \%$ de ellos el recuento absoluto de neutrófilos era inferior a $1500 \mathrm{y}$ en $61,9 \%$ inferior a $500(26 / 42)$. En $13(30,9 \%)$ de los pacientes con LLA habia fiebre como manifestación inicial, que con una sola excepción coincidió con neutropenia severa o extrema. En $9(75 \%)$ de los 12 niños con fiebre y leucopenia severa o extrema se identifico un foco infeccioso, que en seis era respiratorio $y$ en tres se asoció a hemocultivos positivos (25\%) a $S t a-$ phylococcus dorado (dos casos de bronconeumonía) y Klebsiella (un paciente con flegmón de labio superior). La fiebre como único signo se presentó en tres casos (25\%). Este grupo fue tratado con una combinación de antibióticos determinada por una norma local y se modificó según los resultados microbiológicos cuando fue oportuno, con una letalidad de $8,3 \%$ (1/12). Los pacientes con neutropenia afebril fueron 14 , en seis se detectó un problema clínico específico, en tres de ellos los cultivos fueron positivos (dos infecciones urinarias a Escherichia coli y un flegmón de un labio mayor a Pseudomonas aeruginosa). En este grupo sólo se usaron los antibióticos habitualmente reconmendados para cada situación y según antibiograma, con buena respuesta en todos. La inducción de la remisión se inició en la mayoría antes de los 10 días de evolución.

(Palabras claves: Leucemia linfoide aguda, leucopenia severa inicial, infecciones, tratamiento.)

\section{Referencias}

1. Budey GP, Buckley $M$, Sathey $S$ et al.: Quantitative relationship between circulating leucocytes and infections in patients acute leukemia. Ann Intern Med 1966;64: 328-340.

2. Pizzo PA, Commers 1 , Cotton $L$ et al.: Aproaching the controversies in antibacterial management of cancer patients, Am J Med 1984; 76: 436-449.

3. Bishop JF, Schimplf $S C$, Digap CH et al.: Infections during intensive chemotherapy for acute leukemia. Ann Intern Med 1981 ; 95 : 549-555. 
4. Aisner $J$, Murillo $J$. Schimpff $T$ et ol.: Invasive Aspergillosis in acute leukemia. Ann Intern Med $1979 ; 90: 4-9$.

5. Alhano E, Pizzo PA: Infections Complications in childhood with acute leukemia. Pediatr Clin North Am 1988; $35: 873-901$.

6. Schimpff $S C$, Young $V M$, Grene $W H$ et al.: Origin of infections in acute non-lymphocytic leukemia, significance of hospital acquisition of potential pathogens. Ann Intern Med 1972; 77: 70?-714.

7. Sohanson $W G$, Woods $D E$, Chandhesi $T$ : Association of respiratory tract. Colonization with ad herence of Gram nagative bacili to epithelial cells. $\mathrm{J}$ Infect Dis $1979 ; 139 ; 667-673$.

8. Wathorn $J W$, Pizzo PA: Infection complication in the pediatric cancer patient. En: Pizzo PA, Poplack D ed. Pediatric Oncology, Philadelphia. JB Lipim cott 1989:837-869.

9. Hiemenz I, Skelton J, Pizzo PA: Perspective on the management of catheter related infections in cancer patients. Pediatr Infect Dis J 1986; 5: 6-11.

10. Wintrobe $M$ ed: Clinical Hematology Philadelphia: Lea and Febiger, 1981: 1507-1535.

11. Vargas $L$, Pino $S$, Barrio $M$, Young $T$. Garcia $H$ : Aspectos clínicos y hematológicos iniciales de la lewcemia en el niño. Rew Chil Pediatr 1984; 55: 149-154,

12. Miller DR: Acute lymphoblastic letıkemia, Pediatr Clir North Am 1980; 27: 269-291.

13. Pizzo PA, Robichand $K J$. Wesley $R$ et al.: Fever in the pediatric and young adult patient with cancer, a prospective study of 1001 apisodes. Medicine 1982;61:153-165.

14. Pizzo $P A$. Infections complications in the child with cancer Pathophysiology of the compromised host and the initial evaluation and management of the febril patient. J Pediatr 1981; 98: 341-353.

15. Wade $J C$ : Principles of empiric usage in febril patients with granulocytopenia. I. Antinicrob Chemotherapy 1982:9 (Sup A) 215-222.

15. Salgato C. Recker $A$, Camphell $M$ et al: Acute Lymphoblastic leukemia. Febrile episodes in 60 patients in chilean national protocol 1987. Am J Pediatr Hernatal Oncal 1990 12:238 (Abstract).

17. Boder GP, Yadeja 1, Eiring L: Pseudomonas bacteremia: retrospective analysis of $4 t 0$ episodes. Arch Intern Med 1985; 1 Is 1621-1629.

18. Stickles EA, Greene WH. Wiernik PH: Clinicals presentation of infections in granulocytopenia patients. Intern Med 1975; 135: 715-719.

19. Bryant $R E$, Hood $A F$, Hood $C E$ et al. Factors affecting mortality of Gram negative bacteremia. Arch Intern Med 1975;135: 715-719.

20. Pizzo PA. Infection complications in the child with cancet. Management of specific infections organism J Pediatr 19B1:98: 513-523.

21. Louria $D B$ : Infections complications of neoplastic disease. Am J Med 1984; 76:414-420.

22. Pizzo $P A$, Hathorn $U$, Hiemenz $J$ et al.: A randomized trial comparing ceftazidine alone with combination antioiotic therapy in cancer patients with fever and neutropenia. N Eng J Med 1986: $315: 552-558$. 